\section{Response to Cederbaum}

To the Editor: We appreciate the opportunity to respond to the Letter to the Editor by Dr Cederbaum ${ }^{1}$ regarding our publication providing standards and guidelines for the interpretation of sequence variants. ${ }^{2}$ Dr Cederbaum notes that "missense changes in the relevant genes... [are] ... usually called...mutations on the basis of prior probability, the absence of the change as a known polymorphism in the 1000 Genomes Project, knowledge of the severity/significance of the amino acid change to protein structure (like the mutation prediction programs), and the evolutionary conservation of the amino acid affected." We believe that, even taken together, these criteria are insufficient to conclude that a variant causes disease and are likely the basis for erroneous interpretations of variants in the published literature and databases. The assumption that the absence of a variant in a database implies a high likelihood of pathogenicity is incorrect, given that each individual has thousands of unique variants, very few of which cause disease. In addition, prediction algorithms that use evolutionary conservation and biochemical and protein structural data to predict functional damage result in a significant rate of false positives and false negatives. ${ }^{3}$

Dr Cederbaum ${ }^{1}$ raises the issue of "prior probability," which we understand to mean that the patient's phenotype is believed to be associated with a specific gene (or genes). While a single-gene test finding of a novel variant in CFTR may be given additional weight in a child with a positive sweat test, the same would not hold for a gene in a child with a broad phenotype such as autism. Our guideline recognizes that clinical presentation can be useful, but given a wide range of phenotypes with a nonspecific association with genes, this evidence must not be overweighted. Our guideline was developed as an educational resource for clinical laboratories to increase the consistency of variant classification. The approach described in our guideline is meant to be applicable to variants in all Mendelian genes, whether identified by single-gene tests, multigene panels, exome sequencing, or genome sequencing. Of the $~ 5,000$ genes known today to be associated with Mendelian disease, the amount of phenotype information and the characterization of their mutational spectrum are often minimal, necessitating care when interpreting variants in these genes.

We should also note that, although our guideline focused on the laboratory interpretation step, we acknowledge that the practicing physician may have access to additional clinical information, providing the ability to further determine the relationship between reported variants and phenotype. We encourage clinicians to provide as much clinical data as possible to the laboratory when sending testing, and to relay additional information back to the laboratory after testing has been performed. This communication will ensure the most informative interpretative process for improving the accuracy of variant interpretation for all patients.

Dr Cederbaum" says that "No effort was made to suggest an algorithm or a statistical calculation by which one could use prior probability, frequency of mutations among the general populations in the databases, and the frequency of the rare disorder among the population to determine a higher degree of probability." Although we agree that increasing the use of statistically valid approaches are anticipated to improve the probabilistic assessment of pathogenicity, few such models exist today, and therefore we were unable to incorporate these into a general recommendation. We encourage specialty groups to develop more focused guidance for specific genes, given that the applicability and weight assigned to certain criteria may vary by gene and disease. Similarly, we have stated that the weights of each evidence criterion can be increased or decreased if additional information is available. A statistical model has been developed for variant classification of $B R C A 1$ and $B R C A 2$ by the ENIGMA group using a combined likelihood ratio model; however, it takes into account stronger types of evidence beyond those stated by Dr Cederbaum, including segregation data, family history data, case-control statistical comparisons, and co-occurrence data, all from large aggregated data sets. ${ }^{4}$ Unfortunately, such models and large data sets are currently unavailable for most genes/diseases.

We add a final word of caution to Dr Cederbaum's ${ }^{1}$ proposition that "If these conservative, risk-averse standards are applied to the patients whom we see with a high prior probability, a large minority - if not a majority — would receive ambiguous results, rendering the study virtually useless." It is critical to recognize that genetic information is being used to predict disease risk and make life-altering decisions about surgical procedures, expensive therapies, and pregnancy termination. While a physician may have the advantage of knowing how information may be used and can therefore adjust recommendations given an assessment of the severity of an adverse outcome, the laboratory often does not have this context. We believe that variant interpretations should be consistent, based on evidence, and agnostic to the way that the information may be used in patient management, which often cannot be predicted in advance. For example, a physician may choose to suggest a low-risk screening procedure based on a variant of uncertain significance in $B R C A 1$ along with other relevant clinical information and not suggest a prophylactic mastectomy for such a finding.

In conclusion, we support the use of rigorous and consistent standards for variant classification as defined in our guidelines and emphasize that this approach is necessary to improve our knowledge base to a new standard commensurate with the reliable use of genetic information in the practice of medicine. We encourage the development of statistically valid approaches to variant classification that are enhanced by disease-specific knowledge and robust clinical data sets. 
We agree with Dr Cederbaum that "the perfect is the enemy of the good," but satisfaction with "the good" should not inhibit our efforts to improve and perfect the use of genomics in the care of our patients.

\section{DISCLOSURE}

All workgroup members are clinical service providers. No commercial conflict of interest was reported for Sue Richards, David Bick, Soma Das, Wayne Grody, Elaine Spector, Julie Gastier-Foster, Nazneen Aziz, and Karl Voelkerding. The following workgroup members have a commercial conflict of interest: Sherri Bale (GeneDx, BioReference (stock), Advisory boards for RainDance, Ingenuity); Madhuri Hegde (Advisor for: Oxford Genetic Technologies, Tessarae, Ingenuity/Qiagen); Elaine Lyon (Advisory board for Complete Genomics); and Heidi Rehm (Scientific advisory boards: Ingenuity/Qiagen, Complete Genomics, Knome, Focused Genomics).

\section{The Interpretation of Sequence Variants Workgroup}

Department of Molecular and Medical Genetics, Knight Diagnostic Laboratories, Oregon Health \& Science University, Portland, Oregon, USA. Corresponding author: Carolyn Sue Richards, PhD (richarsu@ohsu.edu)

\section{REFERENCES}

1. Cederbaum S. Interpreting sequence variants in a clinical context. Genet Med 2015; 17:1012.

2. Richards S, Aziz N, Bale S, et al. Standards and guidelines for the interpretation of sequence variants: a joint consensus recommendation of the American College of Medical Genetics and Genomics and the Association for Molecular Pathology. Genet Med 2015;17:405-424.

3. Thusberg J, Olatubosun A, Vihinen M. Performance of mutation pathogenicity prediction methods on missense variants. Hum Mutat 2011;32:358-368.

4. Goldgar DE, Easton DF, Byrnes GB, et al. Genetic evidence and integration of various data sources for classifying uncertain variants into a single model. Hum Mutat 200829:1265-1272.

Advance online publication 12 November 2015. doi:10.1038/gim.2015.151 